\title{
Vitamin D and Depressive Symptoms in Adults with Multiple Sclerosis: A Scoping Review
}

\author{
Carmen Concerto ${ }^{1, *(\mathbb{D}}$, Alessandro Rodolico ${ }^{1}{ }^{\mathbb{D}}$, Alessia Ciancio $\left.{ }^{1}{ }^{(}\right)$, Christian Messina ${ }^{2}$, Antimo Natale ${ }^{1}$, \\ Ludovico Mineo $^{1}$, Fortunato Battaglia ${ }^{3}$ (D) and Eugenio Aguglia ${ }^{1}$ (D) \\ 1 Psychiatry Unit, Department of Clinical and Experimental Medicine, University of Catania, \\ 95123 Catania, Italy; alessandro.rodolico@me.com (A.R.); alessia.ciancio@gmail.com (A.C.); \\ antimo.natale@yahoo.it (A.N.); ludwig.mineo@gmail.com (L.M.); eugenio.aguglia@unict.it (E.A.) \\ 2 MS Center, Department “G.F. Ingrassia”, University of Catania, Via Santa Sofia 78, 95123 Catania, Italy; \\ chry.messina@gmail.com \\ 3 Department of Medical Sciences, Neurology and Psychiatry, Hackensack Meridian School of Medicine, \\ Nutley, NJ 07110, USA; fortunato.battaglia@hmhn.org \\ * Correspondence: c.concerto@policlinico.unict.it
}

check for updates

Citation: Concerto, C.; Rodolico, A.; Ciancio, A.; Messina, C.; Natale, A.; Mineo, L.; Battaglia, F.; Aguglia, E. Vitamin D and Depressive Symptoms in Adults with Multiple Sclerosis: A Scoping Review. Int. J. Environ. Res. Public Health 2022, 19, 199. https:// doi.org/10.3390/ijerph19010199

Academic Editor: Paul B. Tchounwou

Received: 27 October 2021

Accepted: 22 December 2021

Published: 25 December 2021

Publisher's Note: MDPI stays neutral with regard to jurisdictional claims in published maps and institutional affiliations.

Copyright: (C) 2021 by the authors. Licensee MDPI, Basel, Switzerland. This article is an open access article distributed under the terms and conditions of the Creative Commons Attribution (CC BY) license (https:// creativecommons.org/licenses/by/ $4.0 /)$.

\begin{abstract}
Background. Vitamin D deficiency has been correlated with Multiple Sclerosis (MS) risk and disease activity. There is some controversy as to whether vitamin D could have an impact on depressive symptoms in people with MS (pwMS). The aim of this scoping review was to evaluate the association between vitamin D status and depressive symptoms in pwMS. Methods. We searched databases to include studies published up to March 2021 to provide an overview of the available evidence on the correlation between vitamin D status and depressive symptoms in pwMS. The eligibility criteria were as follows: studies evaluating the use of vitamin D measurement on depressive symptoms in patients suffering from MS, including randomized and non-randomized studies; studies written in English; and studies exploring an adult population over the age of 18. Results. Eleven studies met our inclusion criteria: two of them were abstracts only; the majority were cross-sectional studies; two were prospective longitudinal studies; one was a retrospective cohort study; and one was a randomized placebo-controlled trial (RCT). Of the eleven studies selected, seven showed a potential correlation between low vitamin D levels and depressive symptoms. Conclusion. Future RCT studies should include patients with greater severity of depressive symptoms and should consider confounding factors such as sun exposure and seasonal variation of vitamin D.
\end{abstract}

Keywords: depression; Multiple Sclerosis; vitamin D

\section{Introduction}

Multiple Sclerosis (MS) is a neurodegenerative disease of the central nervous system (CNS), characterized by demyelination and axonal loss. Evidence from several sources indicates that it is associated with a complex interplay of genetic, immunologic, and environmental factors [1]. According to the most recent studies, about 2.8 million people worldwide have received a diagnosis of MS. MS typically affects young and middle-aged adults with a strong impact on general life functioning [2]. Late-onset MS (LOMS) may occur, albeit even if less frequently, with an estimated prevalence of about $10-20 \%$ of MS cases [3]. MS is clinically characterized by extreme inter and intra-variability in terms of clinical course with different clinical manifestations such as changes in sensation, vision, mobility, balance, and cognition. Based on the initial disease course, MS is classified as either relapsing-remitting MS (RRMS) or primary progressive MS (PPMS) [4,5]. The most frequent clinical course is RRMS, which accounts for approximately $80-85 \%$ of the initial diagnoses and is characterized by relapses followed by periods of remission [5]. People with MS (pwMS) may experience numerous symptoms, including spasticity, fatigue, cognitive dysfunction, depression, bladder dysfunction, bowel dysfunction, sexual dysfunction, and pain [6-9]. Indeed, MS has a multidimensional impact on personal life with symptoms that 
imply a significant loss of autonomy and may increase the risk of psychiatric illness [10]. Depression is one of the most common psychiatric illnesses among pwMS, with a lifetime prevalence estimated to be around 50\% [11,12]. The symptoms may include loss of interest in daily activities, sadness, feelings of helplessness and hopelessness, concentration problems, sleep changes, and loss of energy [13,14]. Moreover, MS management may require lifelong pharmacological interventions $[15,16]$ with different safety concerns due to the lifelong therapy $[17,18]$. The worsening of depressive symptoms can reduce the compliance of patients who take disease-modifying treatments (DMTs) [19]. Psychological and pharmacological approaches have been suggested for the treatment of depression in pwMS with evidence showing a widespread use of antidepressant medications and a clear need for further investigation [12].

Recently, attention has been given to non-pharmacological treatment for the maagement of depression in MS, focusing on Complementary and Alternative Medications (CAMs) [20-22]. CAM is a heterogeneous group of treatments that includes herbal therapies and dietary supplements, previously studied in healthy individuals [23-25] and in pwMS $[20,21,25]$. Among the CAM, attention has been given to vitamin D, a lipidsoluble vitamin mostly produced from exposure of the skin to sunlight; it is also acquired via dietary intake. The metabolite of vitamin $\mathrm{D}$ that is abundant in the circulation is 25-hydroxyvitamin D $(25(\mathrm{OH}) \mathrm{D})$; it is also considered to better describe vitamin D status [26].Vitamin D deficiency is currently considered to be one of the environmental risk factors for the pathogenesis and progression of MS [27]. Vitamin D presents an immunemodulatory effect able to induce an increase in anti-inflammatory cytokines and a decrease in pro-inflammatory cytokines [28]. Previous studies have shown that vitamin D might act in MS by stimulating interleukin (IL)-10 cytokine levels and by reducing IL-17 cytokine levels and the B-cell immunoreactivity [27]. Indeed, it has a favorable impact on the inflammatory pathways of MS and in pwMS; moreover, high circulating levels of vitamin D have been associated with lower risk of disease [20,29].

Vitamin D deficiency has been considered a risk factor for the presence and severity of depressive symptoms in both depressed patients and those with pwMS [30-33]. In depressed patients, increased levels of pro-inflammatory cytokines level-tumor necrosis factor alpha (TNF $\alpha$ ), interleukin (IL)-1 and IL-6- have been reported in the CNS and peripheral circulation $[12,34]$. Previous studies have shown a beneficial effect of vitamin D supplementation on depressive symptoms, supporting the idea that a poor vitamin D status may contribute to depression $[35,36]$. The association of depressive symptoms with vitamin D supplementation or serum vitamin D levels is less established in pwMS [35,37]. Hence, considering the context of the chronic inflammation in MS, vitamin D status and its supplementation have been studied to evaluate the effect on depressive symptoms. The objective of this scoping review was to provide an overview of the available evidence on the correlation between vitamin D status and depressive symptoms in pwMS. In particular, we wanted to answer the following question: Is there an association between vitamin $D$ status and depressive symptoms in pwMS?

\section{Methods}

\subsection{Search Strategy}

In performing this scoping review, we followed the PRISMA-ScR (Preferred Reporting Items for Systematic Reviews and Meta-Analyses Extension for Scoping Reviews) guidelines [38]. A preliminary search for existing scoping reviews (and ideally systematic reviews too) on the topic was conducted. The review included five steps: (1) defining the research question; (2) identifying relevant studies; (3) selecting studies; (4) charting the data; and (5) collecting, summarizing, and reporting the results.

\subsection{Search and Studies Selection}

On 23 March 2021, we conducted systematic searches in MEDLINE (accessed through PubMed), EMBASE, by using the following search string: ("Vitamin D" OR cholecalciferol 
OR colecalciferol OR calcitriol OR 1,25-dihydroxycholecalciferol) AND "multiple sclerosis" AND (depress* OR mood*). The eligibility criteria were as follows: (1) studies evaluating the use of vitamin D measurement on depressive symptoms in patients suffering from MS, including randomized and non-randomized studies; (2) studies written in English; (3) studies exploring an adult population, over the age of 18 . We excluded studies referring to (1) correlation between general depression and vitamin D, with no mention of MS; (2) correlation between general MS symptoms and vitamin D, with no mention of depression. Two independent reviewers (AC and AN) initially screened the titles and abstracts, and then selected the studies to be included after checking the full text. In case of disagreement, the final decision was made with the help of a third reviewer (either CC or AR). The interrater agreement in the title/abstract and full text selection phases was fair (kappa for agreement 0.83 and 0.86 , respectively). Consequently, the relevant data were extracted in a predefined form. The following characteristics were collected: first author and year of publication, country where the study was conducted, study design, study aim, study duration, study population characteristics, method used for vitamin D level measurement, main study results, and cofounding factors (i.e., age, sex, degree of disability, fatigue, sun exposure). After double-checking the extracted data, the resulting forms were merged in one comprehensive table, grouped by study design.

\section{Quality Assessment}

The scoping review considered observational (cross-sectional and cohort) studies and interventional studies. We adopted the appropriate quality assessment tool depending on the study design. In particular, we used the original version of the Newcastle-Ottawa scale for cohort studies [39]. an adapted version of the Newcastle-Ottawa scale developed by Herzog and colleagues [40] for cross-sectional studies. We adopted the National Institutes of Health (NIH) quality assessment tool for before-after (Pre-Post) study with no control group, for arm interventional studies [41]. Finally, we assessed the randomized controlled trials quality by using the Cochrane Risk of Bias 2.0 [42].

\section{Results}

The PRISMA flow chart is shown in Figure 1. A total of 95 potentially relevant studies were identified.

After the title screening, 58 were excluded, leaving 37 studies. Of the 37 studies that were screened for eligibility, only 11 were considered eligible and included. Among them, nine were full text articles, two were abstracts, seven were cross-sectional studies [26,43-48] one was a retrospective cohort study [49] and two were prospective longitudinal studies [50,51]. Only one study was a RCT [52]. The included studies were conducted in various countries (Iran, Portugal, Argentina, Romania, Jordan, Australia, the Netherlands, and Saudi Arabia). The earliest publication date was 2012 and the most recent was 2021. Most of the studies assessed a population of patients with RRMS. Some studies explored if there was a correlation between vitamin D serum level -defined as vitamin D status-and depressive symptoms, meanwhile other studies reported the effect of vitamin D supplementation on depression in MS. Table 1 presents a detailed summary of the core characteristics of the included studies. 


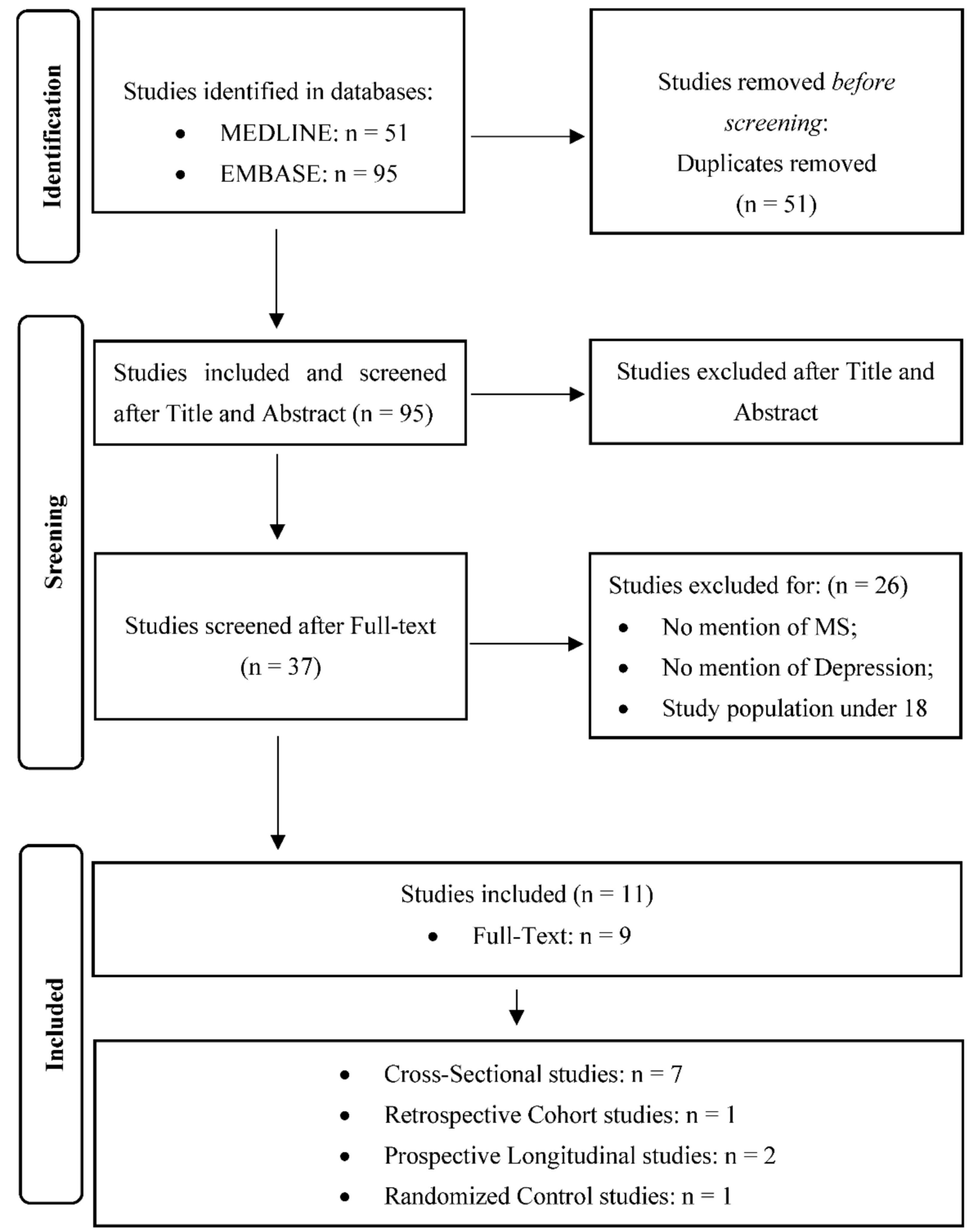

Figure 1. PRISMA flowchart. 
Table 1. Included studies.

\begin{tabular}{|c|c|c|c|c|}
\hline $\begin{array}{c}\text { Author, } \\
\text { Year (Country) } \\
\text { Study Design (D) } \\
\text { Recruitment Timing (RT ) } \\
\text { Followup (FU) } \\
\text { Study Population Characteristics }\end{array}$ & Vitamin D Measurements & $\begin{array}{l}\text { Depression } \\
\text { Measuremnts }\end{array}$ & $\begin{array}{l}\text { Other Measured } \\
\text { Variables }\end{array}$ & $\begin{array}{c}\text { Study Aim(s) } \\
\text { Results } \\
\text { Cofounder } \\
\text { Quality Assessment }\end{array}$ \\
\hline $\begin{array}{l}\text { Ashtari F. et al., } 2013 \text { (Iran) } \\
\text { D: Cross-sectional study } \\
\text { RT: March } 2011 \text { to September } 2011 \\
\text { FU: NR } \\
\text { Participants: } 200 \\
\text { Mean Age (SD): } 33.5 \text { (8.4) Female/Male: } \\
\text { 154/46 } \\
\text { MS Diagnosis: McDonald's criteria } \\
\text { Clinical status: } 94 \% \text { of patients have a } \\
\text { RR MS, relapse free for more than } \\
8 \text { weeks prior to the study selected. }\end{array}$ & $\begin{array}{l}\text { Analyzed fluid: venous blood } \\
\text { Analythical methodology: } \\
\text { radioimmunoassay (RIA) method } \\
\text { using Biosource kit } \\
\text { Vit. D concentration (nmol/L): } 76 \\
\text { (IQR: } 35.5-100.7) \\
\text { Vit. D status: } 51.5 \% \text { normal vit. D level } \\
\text { (>75 mmol/L), } 49.5 \% \text { low vit. D level } \\
\text { (<75 mmol/L) Dietary vit. D intake: } \\
\text { NA }\end{array}$ & $\begin{array}{l}\text { Evaluation of } \\
\text { depressive symptoms: } \\
\text { Beck Depression } \\
\text { Inventory for Primary } \\
\text { Care (BDI-PC) } \\
\text { Depressive symptoms } \\
\text { severity: } 4 \text { (3-7) in the } \\
\text { overall sample }\end{array}$ & $\begin{array}{l}\text { Sun exposure: Not } \\
\text { Measured } \\
\text { Fatigue Diagnosis: } \\
\text { Fatigue Severity Scale } \\
\text { (FFS) } \\
\text { Degree of disability: } \\
\text { Expanded Disability } \\
\text { Status Score (EDSS) }\end{array}$ & $\begin{array}{l}\text { Aim: To investigate the relation between vitamin D status with fatigue and } \\
\text { depressive symptoms } \\
\text { Results: Vitamin D status was inversely associated with depressive symptoms. } \\
\text { Effect size: correlation, } \beta=-0.16, p \text {-value }=0.02 \\
\text { Controlled cofounders: not considered } \\
\text { Quality asessment (NOS—cross sectional studies adaptation):Selection (max } \\
\text { 5): } 3 \\
-\quad \text { Representativenes: } 1 \\
-\quad \text { Sample size: } 0 \\
\text { - Non-respondents: } 0 \\
\text { - } \quad \text { Exposure: } 2 \\
\text { Comparability (max 2): } 0 \\
\text { Outcome (max 3): } 2 \\
\text { - } \quad \text { Assesment: } 1 \\
\text { - Statistical test: } 1 \\
\text { Total score (max } 10): 5\end{array}$ \\
\hline $\begin{array}{l}\text { Knippenberg et al., } 2011 \text { (The } \\
\text { Netherlands) } \\
\text { D: Cross-sectional study } \\
\text { RT: 2005-2007 } \\
\text { FU: NR } \\
\text { Participants: } 59 \\
\text { Mean Age (SD): } 44.2 \text { (9.2) } \\
\text { Female/Male: } 16 / 43 \\
\text { MS Diagnosis: McDonald's criteria } \\
\text { Clinical status: Participants had not used } \\
\text { corticosteroids for } \geq 4 \text { weeks and were } \\
\text { relapse free for } \geq 4 \text { weeks prior to } \\
\text { assessment. }\end{array}$ & $\begin{array}{l}\text { Analyzed fluid: serum } \\
\text { Analythical methodology: NA } \\
\text { Vit. D concentration (nmol/L): } 62.3 \\
\text { (SD 27.8) } \\
\text { Vit. D status: } 23 \% \text { normal vit. D level } \\
\text { (>75 mmol/L), } 73 \% \text { low vit. D level } \\
\text { (<75 mmol/L) } \\
\text { Dietary vit. D intake: NA }\end{array}$ & $\begin{array}{l}\text { Evaluation of } \\
\text { depressive symptoms: } \\
\text { Hospital Anxiety and } \\
\text { Depression Scale, } \\
\text { depression subscale } \\
\text { (HADS-D) } \\
\text { Depressive symptoms } \\
\text { severity: } 6.2 \text { (SD 4.4) }\end{array}$ & $\begin{array}{l}\text { Sun exposure: Not } \\
\text { Measured } \\
\text { Fatigue Diagnosis: } \\
\text { Multidimensional } \\
\text { Fatigue Inventory (MFI) } \\
\text { Degree of disability: } \\
\text { Expanded Disability } \\
\text { Status Score (EDSS) }\end{array}$ & $\begin{array}{l}\text { Aim: To assess whether vitamin D status contributes to the presence of } \\
\text { depressive symptoms and fatigue in MS. } \\
\text { Results: Vitamin D status correlated negatively with depression. In a multiple } \\
\text { regression model, vitamin D status was not a significant contributor to } \\
\text { depression. } \\
\text { Effect size: correlation, } \beta=-0.33, p \text {-value }=0.006 \\
\text { Controlled cofounders: age, EDSS and MFI scors } \\
\text { Quality asessment (NOS-cross sectional studies adaptation): } \\
\text { Selection (max 5): } 2 \\
\text { - } \quad \text { Representativenes: } 0 \\
\text { - Sample size: } 0 \\
\text { - Non-respondents: } 0 \\
\text { - Exposure: } 2 \\
\text { Comparability (max 2): } 1 \\
\text { Outcome (max 3): } 2 \\
\text { - } \quad \text { Assesment: } 1 \\
\text { Statistical test: } 1 \\
\text { Total score (max } 10): 5\end{array}$ \\
\hline
\end{tabular}


Table 1. Cont.

\begin{tabular}{|c|c|c|c|c|}
\hline $\begin{array}{c}\text { Author, } \\
\text { Year (Country) } \\
\text { Study Design (D) } \\
\text { Recruitment Timing (RT ) } \\
\text { Followup (FU) } \\
\text { Study Population Characteristics }\end{array}$ & Vitamin D Measurements & $\begin{array}{l}\text { Depression } \\
\text { Measuremnts }\end{array}$ & $\begin{array}{l}\text { Other Measured } \\
\text { Variables }\end{array}$ & $\begin{array}{c}\text { Study Aim(s) } \\
\text { Results } \\
\text { Cofounder } \\
\text { Quality Assessment }\end{array}$ \\
\hline $\begin{array}{l}\text { Silva et al., } 2016 \text { * (Argentina) } \\
\text { D: Cross-sectional study RT: NA } \\
\text { FU: NR } \\
\text { Participants: } 61 \\
\text { Mean Age (SD): } 42.8 \text { (12.4) } \\
\text { Female/Male: } 44 / 17 \\
\text { MS Diagnosis: NA } \\
\text { Clinical Status: Relapsing Remitting } \\
\text { MS (RRMS). }\end{array}$ & $\begin{array}{l}\text { Analyzed fluid: serum } \\
\text { Analythical methodology: } \\
\text { chemiluminescence } \\
\text { Vit. D mean (SD) (nmol/L): NA } \\
\text { Vit. D status: } 34 \% \text { vit. D level } \\
\text { ( } \leq 49.92 \mathrm{mmol} / \mathrm{L}), 66 \% \text { low vit. D } \\
\text { level (>49.92 mmol/L) } \\
\text { Dietary vit. D intake: NA }\end{array}$ & $\begin{array}{l}\text { Evaluation of } \\
\text { depressive } \\
\text { symptoms: Beck } \\
\text { Depression } \\
\text { Inventory II (BDI } \\
\text { II). } \\
\text { Depressive } \\
\text { symptoms severity: } \\
\text { NA }\end{array}$ & $\begin{array}{l}\text { Sun exposure: Not } \\
\text { Measured } \\
\text { Neuropsychological } \\
\text { status: Brief } \\
\text { Repeatable } \\
\text { Neuropsychological } \\
\text { Battery (BNS-EM) } \\
\text { Fatigue Diagnosis: } \\
\text { Fatigue Severity } \\
\text { Scale (FSS) } \\
\text { Degree of disability: } \\
\text { Expanded Disability } \\
\text { Status Score (EDSS) }\end{array}$ & $\begin{array}{l}\text { Aim: To evaluate the relationship between serum vitamin D levels } \\
\text { and cognitive impairment, depression and fatigue } \\
\text { Results: [An association between depression severity and vitamin D } \\
\left.\text { levels was observed ( } p<0.01, \mathrm{X}^{2}=30.7\right) \text {. } \\
\text { Effect size: odds ratio not reported } \\
\text { Controlled cofounders: not considered } \\
\text { Quality asessment (NOS—cross sectional studies adaptation): } \\
\text { Selection (max 5): } 2 \\
\text { - } \quad \text { Representativenes: } 0 \\
\text { - } \quad \text { Sample size: } 0 \\
\text { - Non-respondents: } 0 \\
\text { - } \quad \text { Exposure: } 2 \\
\text { Comparability (max } 2): 0 \\
\text { Outcome (max } 3): 3 \\
\text { - Assesment: } 2 \\
\text { - Statistical test: } 1 \\
\text { Total score (max } 10): 5\end{array}$ \\
\hline
\end{tabular}


Table 1. Cont.

\begin{tabular}{|c|c|c|c|c|}
\hline $\begin{array}{c}\text { Author, } \\
\text { Year (Country) } \\
\text { Study Design (D) } \\
\text { Recruitment Timing (RT ) } \\
\text { Followup (FU) } \\
\text { Study Population Characteristics }\end{array}$ & Vitamin D Measurements & $\begin{array}{l}\text { Depression } \\
\text { Measuremnts }\end{array}$ & $\begin{array}{c}\text { Other Measured } \\
\text { Variables }\end{array}$ & $\begin{array}{c}\text { Study Aim(s) } \\
\text { Results } \\
\text { Cofounder } \\
\text { Quality Assessment }\end{array}$ \\
\hline $\begin{array}{l}\text { Freitas et al., } 2017 \text { (Portugal) } \\
\text { D: Cross-sectional study } \\
\text { RT: May 2016-January } 2017 \\
\text { FU: NR } \\
\text { Participants: } 54 \text { (78 study } \\
\text { participants, } 24 \text { without vit. D data) } \\
\text { Mean Age: NA } \\
\text { Female/Male: NA } \\
\text { MS Diagnosis: McDonald's criteria } \\
\text { Clinical Status: } 55 \% \text { RR MS. }\end{array}$ & $\begin{array}{l}\text { Analyzed fluid: serum } \\
\text { Analythical methodology: NA } \\
\text { Vit. D mean (SD) (nmol/L): } 78.7 \\
\text { (SD 48.9) } \\
\text { Vit. D status: } 27.8 \% \text { vit. D level } \\
\text { deficiency ( } \leq 49.92 \mathrm{mmol} / \mathrm{L}) \text {, } \\
42.6 \% \text { low vit. D level } \\
(49.92-72.4 \mathrm{mmol} / \mathrm{L}), 29.6 \% \\
\text { normal vit. D levels } \\
\text { (>72.4 mmol/L) } \\
\text { Dietary vit. D intake: } 34.6 \% \text { were } \\
\text { taking vit. D continuing } \\
\text { supplement }\end{array}$ & $\begin{array}{l}\text { Evaluation of } \\
\text { depressive } \\
\text { symptoms: } \\
\text { Hospital Anxiety } \\
\text { and Depression } \\
\text { questionnaire } \\
\text { (HADS-D) } \\
\text { Depressive } \\
\text { symptoms severity: } \\
\text { NA }\end{array}$ & $\begin{array}{l}\text { Sun exposure: recall } \\
\text { questionnaire } \\
\text { assessing daily time } \\
\text { in sun and skin } \\
\text { exposure (face, limbs, } \\
\text { body) for the } \\
\text { previous week } \\
\text { Fatigue Diagnosis: } \\
\text { Modified Fatigue } \\
\text { Impact Scale (MFIS) } \\
\text { Daytime sleepiness: } \\
\text { Epworth Sleepiness } \\
\text { Scale (ESS) } \\
\text { Degree of disability: } \\
\text { Expanded Disability } \\
\text { Status Score (EDSS) }\end{array}$ & $\begin{array}{l}\text { Aim: To evaluate the prevalence and severity of fatigue and its } \\
\text { relationship with other clinical variables. } \\
\text { Results: No association between } 25-O H-D \text { levels and depression. } \\
\text { Effect size: correlation not reported } \\
\text { Controlled cofounders: not considered } \\
\text { Quality asessment (NOS—cross sectional studies adaptation): } \\
\text { Selection (max 5): } 2 \\
\text { - } \quad \text { Representativenes: } 0 \\
\text { - } \quad \text { Sample size: } 0 \\
\text { - Non-respondents: } 0 \\
\text { - } \quad \text { Exposure: } 2 \\
\text { Comparability (max 2): } 0 \\
\text { Outcome (max 3): } 1 \\
\text { - } \quad \text { Assesment: } 1 \\
\text { - Statistical test: } 0 \\
\text { Total score (max } 10): 3\end{array}$ \\
\hline
\end{tabular}


Table 1. Cont.

\begin{tabular}{|c|c|c|c|c|}
\hline $\begin{array}{c}\text { Author, } \\
\text { Year (Country) } \\
\text { Study Design (D) } \\
\text { Recruitment Timing (RT ) } \\
\text { Followup (FU) } \\
\text { Study Population Characteristics }\end{array}$ & Vitamin D Measurements & $\begin{array}{l}\text { Depression } \\
\text { Measuremnts }\end{array}$ & $\begin{array}{l}\text { Other Measured } \\
\text { Variables }\end{array}$ & $\begin{array}{c}\text { Study Aim(s) } \\
\text { Results } \\
\text { Cofounder } \\
\text { Quality Assessment }\end{array}$ \\
\hline $\begin{array}{l}\text { Tiu et al., } 2017 * \text { (Romania) } \\
\text { D: Cross-sectional Study } \\
\text { RT: NA } \\
\text { FU: NR } \\
\text { Participants: } 106 \\
\text { Mean Age (SD): } 38.7(10.1) \\
\text { Female/Male: } 72 / 34 \\
\text { Clinical Status: patients treated with } \\
\text { immunomodulatory drugs. }\end{array}$ & $\begin{array}{l}\text { Analyzed fluid: serum } \\
\text { Analythical methodology: } \\
\text { chemiluminescence } \\
\text { Vit. D mean (SD) (nmol/L): } 57.9 \\
\text { (SD 29.5) } \\
\text { Vit. D status: } 81.1 \% \text { vit. D level } \\
\text { deficiency ( } \leq 74.9 \text { mmol/L) } \\
\text { Dietary vit. D intake: NA }\end{array}$ & $\begin{array}{l}\text { Evaluation of } \\
\text { depressive } \\
\text { symptoms: Beck } \\
\text { Depression } \\
\text { Inventory for } \\
\text { Primary Care } \\
\text { (BDI-PC) } \\
\text { Depressive } \\
\text { symptoms severity: } \\
\text { NA }\end{array}$ & $\begin{array}{l}\text { Sun exposure: Not } \\
\text { Measured }\end{array}$ & $\begin{array}{l}\text { Aim: To investigate the relationship between vitamin D status, } \\
\text { depression and self-reported impact of disease } \\
\text { Results: no significant association between low vitamin D levels and } \\
\text { depression } \\
\text { Effect size: odds ratio not reported } \\
\text { Controlled cofounders: not considered } \\
\text { Quality asessment (NOS—cross sectional studies adaptation): } \\
\text { Selection (max 5): } \\
\text { - } \quad \text { Representativenes: } 0 \\
\text { - } \quad \text { Sample size: } 0 \\
\text { - Non-respondents:0 } \\
\text { - } \quad \text { Exposure: } 2 \\
\text { Comparability (max } 2): 0 \\
\text { Outcome (max } 3): 3 \\
\text { - Assesment: } 2 \\
\text { - Statistical test: } 1 \\
\text { Total score (max } 10): 5\end{array}$ \\
\hline
\end{tabular}


Table 1. Cont.

\begin{tabular}{|c|c|c|c|c|}
\hline $\begin{array}{c}\text { Author, } \\
\text { Year (Country) } \\
\text { Study Design (D) } \\
\text { Recruitment Timing (RT ) } \\
\text { Followup (FU) } \\
\text { Study Population Characteristics }\end{array}$ & Vitamin D Measurements & $\begin{array}{l}\text { Depression } \\
\text { Measuremnts }\end{array}$ & $\begin{array}{c}\text { Other Measured } \\
\text { Variables }\end{array}$ & $\begin{array}{c}\text { Study Aim(s) } \\
\text { Results } \\
\text { Cofounder } \\
\text { Quality Assessment }\end{array}$ \\
\hline $\begin{array}{l}\text { El Salem et al., } 2021 \text { (Jordan) } \\
\text { D: Cross-sectional study } \\
\text { RT: October 2018-June } 2019 \\
\text { FU: NR } \\
\text { Participants: } 88 \\
\text { Mean Age (SD): } 36 \text { (10.69) } \\
\text { Female/Male: } 64 \text { /24 } \\
\text { MS Diagnosis: } 2017 \text { revised } \\
\text { McDonald MS diagnostic } \\
\text { Clinical Status: Being relapse-free } \\
\text { for } 30 \text { days prior to participation. }\end{array}$ & $\begin{array}{l}\text { Analyzed fluid: blood } \\
\text { Analythical methodology: } \\
\text { Enzyme-Linked Immunosorbent } \\
\text { Assay (ELISA) } \\
\text { Vit. D mean (SD) (nmol/L): } 60.2 \\
\text { (SD 25.9) } \\
\text { Vit. D status: } 40.9 \% \text { vit. D level } \\
\text { deficiency ( } \leq 49.9 \mathrm{mmol} / \mathrm{L}), 27.3 \% \\
\text { low vit. D level } \\
\text { (49.9-74.9 mmol/L), 31.8\% normal } \\
\text { vit. D levels (>74.9 mmol/L) } \\
\text { Dietary vit. D intake: NA }\end{array}$ & $\begin{array}{l}\text { Evaluation of } \\
\text { depressive } \\
\text { symptoms: } \\
\text { Hospital Anxiety } \\
\text { and Depression } \\
\text { Scale (HADS-D); } \\
\text { Beck Depression } \\
\text { Inventory-II rating } \\
\text { scale(BDI-II) } \\
\text { Depressive } \\
\text { symptoms severity: } \\
\text { HADS-D: } 8.45 \text { (SD } \\
\text { 5.28); BDI-II: } 17.93 \\
\text { (SD 11.82) }\end{array}$ & $\begin{array}{l}\text { Sun exposure: Not } \\
\text { Measured } \\
\text { Degree of disability: } \\
\text { Expanded Disability } \\
\text { Status Score (EDSS) } \\
\text { Perceived level of } \\
\text { motor disability: } \\
\text { Patient-determined } \\
\text { disease steps (PDDS) }\end{array}$ & $\begin{array}{l}\text { Aim: To evaluate vitamin D levels and its correlation with validated } \\
\text { depression scales. } \\
\text { Results: Serum Vit. D levels significantly correlated with scores of } \\
\text { depression scales regardless of sex. The HADS depression score } \\
\text { significantly correlated with serum Vit. D levels. Similarly, a } \\
\text { significant inverse association between BDI-II score and Vit. D level } \\
\text { was noted. } \\
\text { Effect size: Pearson correlation coefficient, } r=-0.513, p<0.001 \text { (for } \\
\text { HADS), } \mathrm{r}=-0.401, p<0.001 \text { (for BDI-II) } \\
\text { Controlled cofounders: age, sex, BMI, duration of disease, type of MS, } \\
\text { and EDSS } \\
\text { Quality asessment (NOS-cross sectional studies adaptation): } \\
\text { Selection (max 5): } 2 \\
\text { - } \\
\text { - Representativenes: } 0 \\
\text { Sample size: } 0 \\
\text { - Non-respondents: } 0 \\
\text { - Exposure: } 2 \\
\text { Comparability (max 2): } 1 \\
\text { Outcome (max 3): } 3 \\
\text { - } \text { Assesment: } 2 \\
\text { - Statistical test: } 1 \\
\text { Total score (max } 10): 6\end{array}$ \\
\hline
\end{tabular}


Table 1. Cont.

\begin{tabular}{|c|c|c|c|c|}
\hline $\begin{array}{c}\text { Author, } \\
\text { Year (Country) } \\
\text { Study Design (D) } \\
\text { Recruitment Timing (RT ) } \\
\text { Followup (FU) } \\
\text { Study Population Characteristics }\end{array}$ & Vitamin D Measurements & $\begin{array}{l}\text { Depression } \\
\text { Measuremnts }\end{array}$ & $\begin{array}{l}\text { Other Measured } \\
\text { Variables }\end{array}$ & $\begin{array}{c}\text { Study Aim(s) } \\
\text { Results } \\
\text { Cofounder } \\
\text { Quality Assessment }\end{array}$ \\
\hline $\begin{array}{l}\text { Taylor et al., } 2014 \text { (Australia) } \\
\text { D: Cross-sectional study } \\
\text { RT: NA } \\
\text { FU: NR } \\
\text { Participants: } 2225 \text { Median Age } \\
\text { (IQR): } 45 \text { (38-53) } \\
\text { Female/Male: } 388 / 1813 \\
\text { MS Diagnosis: self-report } \\
\text { Clinical Status: Being relapse-free } \\
\text { for } 30 \text { days prior to participation, } \\
\text { the majority }(61.3 \%) \text { had RR MS. }\end{array}$ & $\begin{array}{l}\text { Analyzed fluid: not measured } \\
\text { Analythical methodology: NA } \\
\text { Vit. D mean (SD) (nmol/L): NA } \\
\text { Vit. D status: NA } \\
\text { Dietary vit. D intake: } 30.1 \% \text { no } \\
\text { daily vit. D consumption, } 17.4 \% \\
1-5000 \text { IU daily vit. D } \\
\text { consumption, > } 5000 \text { IU vit. D } \\
\text { consumption. }\end{array}$ & $\begin{array}{l}\text { Evaluation of } \\
\text { depressive } \\
\text { symptoms: Patient } \\
\text { Health } \\
\text { Questionnaire } \\
\text { depression module } \\
\text { short version } \\
\text { (PHQ-2) } \\
\text { Depressive } \\
\text { symptoms severity: } \\
\text { NA }\end{array}$ & $\begin{array}{l}\text { Sun exposure: Not } \\
\text { Measured } \\
\text { Fatigue Diagnosis: } \\
\text { The Fatigue Severity } \\
\text { Scale (FSS) } \\
\text { Perceived level of } \\
\text { disability: } \\
\text { Patient-determined } \\
\text { disease steps (PDDS) } \\
\text { Habits Description: } \\
\text { Diet Habits } \\
\text { Questionnaire (DHQ) } \\
\text { removed four items } \\
\text { assessing salt use and } \\
\text { alcohol intake; } \\
\text { International } \\
\text { Physical Activity } \\
\text { Questionnaire (IPAQ) } \\
\text { Comorbidities } \\
\text { Assessment: } \\
\text { Self-Administered } \\
\text { Comorbidity } \\
\text { Questionnaire (SCQ) }\end{array}$ & $\begin{array}{l}\text { Aim: To examine depression and its association with modifiable } \\
\text { lifestyle risk factors } \\
\text { In the lifestyle factors: Vitamin D supplementation. } \\
\text { Results: Taking any vitamin D supplement was associated with lower } \\
\text { odds of screening positive for depression, but taking at least } 5000 \text { IU } \\
\text { daily was associated with the greatest odds. } \\
\text { Effect size: adjusted odds ratio } 0.57 \text { (0.43-0.77) for } 1-5000 \text { IU, } 0.47 \\
(0.32-0.70) \text { for }>5000 \text { IU. } \\
\text { Controlled cofounders: years since diagnosis, number of } \\
\text { comorbidities, level of disability, clinically significant fatigue, age, } \\
\text { gender, marital status and level of education. } \\
\text { Quality asessment (NOS-cross sectional studies adaptation): } \\
\text { Selection (max 5): } 2 \\
\text { - } \quad \text { Representativenes: } 1 \\
\text { - Sample size: } 1 \\
\text { - Non-respondents: } 0 \\
\text { - Exposure: } 0 \\
\text { Comparability (max } 2): 1 \\
\text { Outcome (max } 3): 2 \\
\text { - } \\
\text { Assesment: } 1 \\
\quad \text { Statistical test: } 1 \\
\text { Total score (max } 10): 5\end{array}$ \\
\hline
\end{tabular}


Table 1. Cont.

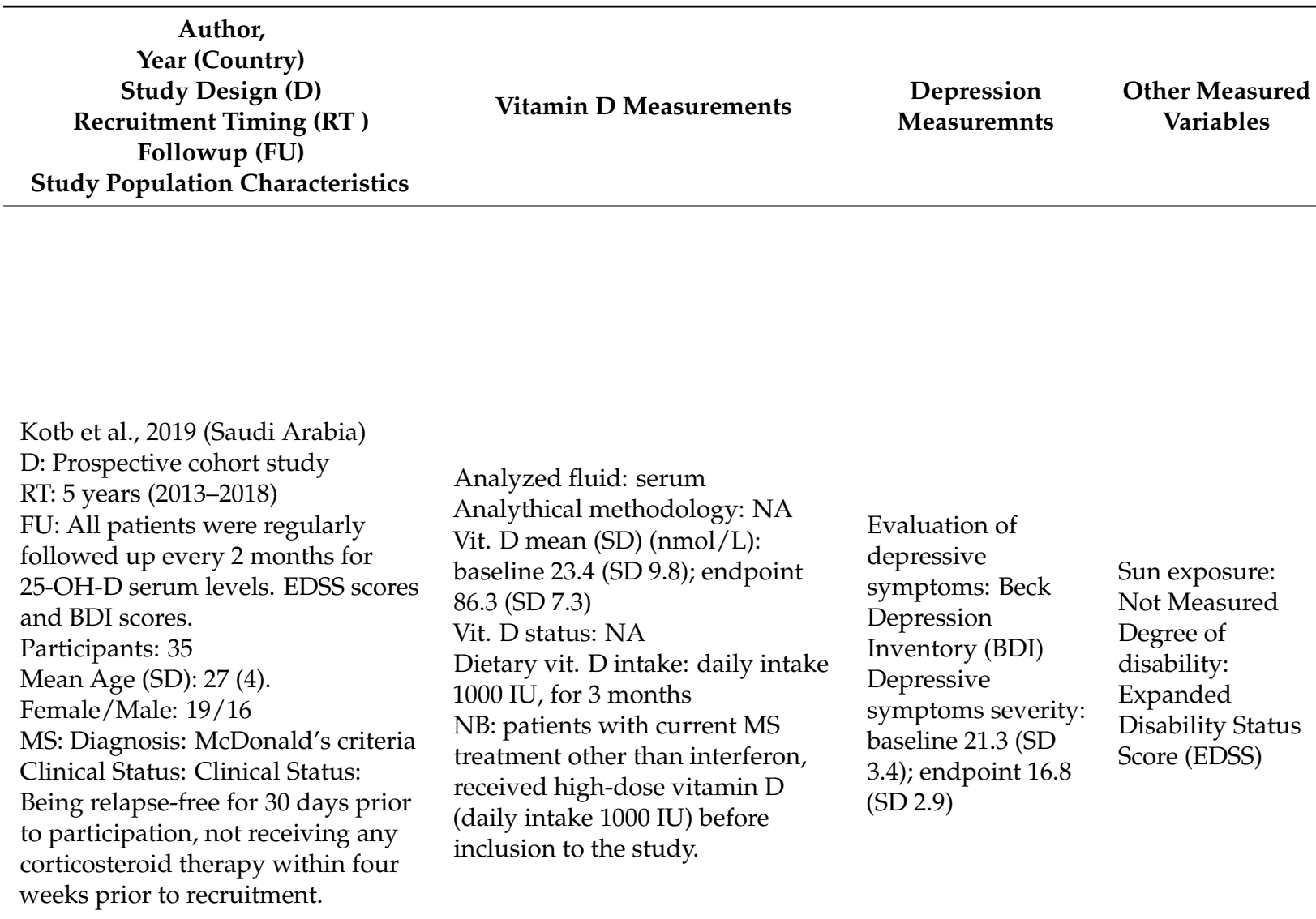

Aim: evaluate the relation between vitamin D levels and depression
scores, and the effect of vitamin D replacement on the depressive symptoms in patients with MS.

Results: A significant negative correlation was observed between vitamin $25(\mathrm{OH}) \mathrm{D}$ levels and Beck's depression inventory scores at baseline $(p<0.001)$, eighth, tenth, and twelfth month $(p=0.001)$.

Effect size: Pearson correlation coefficient $\mathrm{r}=-0.432(p$-value $=0.011)$ Controlled cofounders: EDSS

Quality assessment (NIH quality assessment tool for before-after

(Pre-Post) study with no control group):

1. Was the study question or objective clearly stated? Yes

2. Were eligibility/selection criteria for the study population prespecified and clearly described? Yes

3. Were the participants in the study representative of those who would be eligible for the test/service/intervention in the general or clinical population of interest? Yes

4. Were all eligible participants that met the prespecified entry criteria enrolled? Not reported

5. Was the sample size sufficiently large to provide confidence in the findings? No

6. Was the test/service/intervention clearly described and delivered consistently across the study population? Yes

7. Were the outcome measures prespecified, clearly defined, valid, reliable, and assessed consistently across all study participants? Yes

8. Were the people assessing the outcomes blinded to the participants exposures/interventions? No

9. Was the loss to follow-up after baseline $20 \%$ or less? Were those lost to follow-up accounted for in the analysis? No 
Table 1. Cont.

\begin{tabular}{|c|c|c|c|c|}
\hline $\begin{array}{c}\text { Author, } \\
\text { Year (Country) } \\
\text { Study Design (D) } \\
\text { Recruitment Timing (RT ) } \\
\text { Followup (FU) } \\
\text { Study Population Characteristics }\end{array}$ & $\begin{array}{c}\text { Vitamin D } \\
\text { Measurements }\end{array}$ & $\begin{array}{l}\text { Depression } \\
\text { Measuremnts }\end{array}$ & $\begin{array}{l}\text { Other Measured } \\
\text { Variables }\end{array}$ & $\begin{array}{c}\text { Study Aim(s) } \\
\text { Results } \\
\text { Cofounder } \\
\text { Quality Assessment }\end{array}$ \\
\hline & & & & $\begin{array}{l}10 \text { Did the statistical methods examine changes in outcome measures } \\
\text { from before to after the intervention? Were statistical tests done that } \\
\text { provided p values for the pre-to-post changes? Yes } \\
11 \text { Were outcome measures of interest taken multiple times before the } \\
\text { intervention and multiple times after the intervention (i.e., did they } \\
\text { use an interrupted time-series design)? Yes } \\
12 \text { If the intervention was conducted at a group level (e.g., a whole } \\
\text { hospital, a community, etc.) did the statistical analysis take into } \\
\text { account the use of individual-level data to determine effects at the } \\
\text { group level? Not aplicable }\end{array}$ \\
\hline & & & & Quality Rating: Fair \\
\hline $\begin{array}{l}\text { Taylor et al., } 2018 \text { (Australia) } \\
\text { SD: Retrospective cohort study } \\
\text { RT: NA } \\
\text { FU: } 2.5 \text { years } \\
\text { Participants: } 1401 \\
\text { Mean Age (SD): } 48.4(10.5) \\
\text { Female/Male: } 1150 / 241 \\
\text { Diagnosis MS: } \\
\text { Clinical Status: Being relapse-free } \\
\text { for } 30 \text { days prior to participation, } \\
\text { the majority }(59.3 \%) \text { had RR MS. }\end{array}$ & $\begin{array}{l}\text { Analyzed fluid: NA } \\
\text { Analythical } \\
\text { methodology: NA } \\
\text { Vit. D mean (SD) } \\
\text { (nmol/L): NA } \\
\text { Vit. D status: NA } \\
\text { Dietary vit. D intake: } \\
\text { Vitamin D } \\
\text { supplementation } \\
\text { self-reported. }\end{array}$ & $\begin{array}{l}\text { Evaluation of } \\
\text { depressive } \\
\text { symptoms: patient } \\
\text { Health } \\
\text { Questionnaire-2 } \\
\text { (PHQ-2) at baseline, } \\
\text { and Patient Health } \\
\text { Questionnaire-9 } \\
\text { (PHQ-9) at } \\
\text { follow-up } \\
\text { Depressive } \\
\text { symptoms severity: } \\
\text { NA }\end{array}$ & $\begin{array}{l}\text { Sun exposure: Not Measured } \\
\text { Fatigue Diagnosis: The } \\
\text { Fatigue Severity Scale (FSS) } \\
\text { Perceived level of disability: } \\
\text { Patient-determined disease } \\
\text { steps (PDDS) } \\
\text { Habits Description: Diet } \\
\text { Habits Questionnaire (DHQ) } \\
\text { removed four items assessing } \\
\text { salt use and alcohol intake; } \\
\text { International Physical Activity } \\
\text { Questionnaire (IPAQ) } \\
\text { Severity of disease: Patient } \\
\text { Determined Multiple Sclerosis } \\
\text { Severity Score (P-MSSS) }\end{array}$ & $\begin{array}{l}\text { Aim: Evaluating whether modifiable lifestyle factors were associated } \\
\text { with screening positive for depression } 2.5 \text { years after our baseline study } \\
\text { and the predictors of change in depression screen during follow-up. In } \\
\text { the lifestyle factors: Vit D supplementation. } \\
\text { Results: Vitamin D supplementation was associated with lower } \\
\text { frequencies of depression risk by both PHQ- } 2 \text { and PHQ-9. } \\
\text { Effect size: adjusted odds ratio } 0.70(0.56-0.87)(p \text {-value }=0.002) \\
\text { Controlled cofounders: age, P-MSSS, FSS, and use of antidepressant } \\
\text { medication } \\
\text { Quality assessment (NOS): } \\
\text { Selection (max } 4): 3 \\
\text { - } \quad \text { Representativeness: } 1 \\
\text { - Selection of the non exposed: } 1 \\
\text { - } \quad \text { Ascertainment of exposure: } 1 \\
\text { - Outcome not present at start: } 0 \\
\text { Comparability (max } 2): 1\end{array}$ \\
\hline
\end{tabular}


Table 1. Cont.

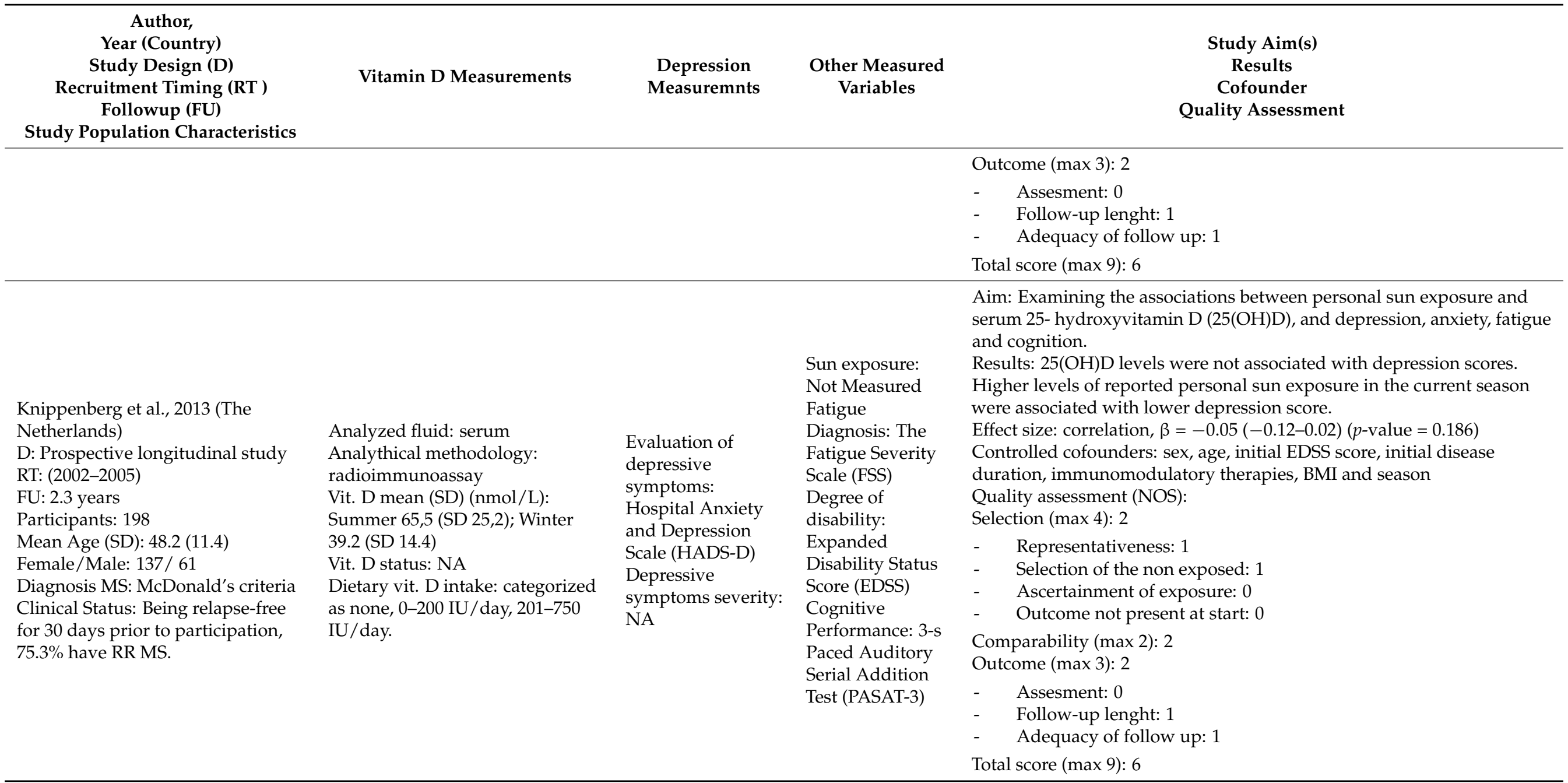


Table 1. Cont.

\begin{tabular}{|c|c|c|c|c|}
\hline $\begin{array}{c}\text { Author, } \\
\text { Year (Country) } \\
\text { Study Design (D) } \\
\text { Recruitment Timing (RT ) } \\
\text { Followup (FU) } \\
\text { Study Population Characteristics }\end{array}$ & Vitamin D Measurements & $\begin{array}{l}\text { Depression } \\
\text { Measuremnts }\end{array}$ & $\begin{array}{c}\text { Other Measured } \\
\text { Variables }\end{array}$ & $\begin{array}{c}\text { Study Aim(s) } \\
\text { Results } \\
\text { Cofounder } \\
\text { Quality Assessment }\end{array}$ \\
\hline $\begin{array}{l}\text { Rolf et al., } 2017 \text { (The Netherlands) } \\
\text { D: Randomized Placebo controlled } \\
\text { trial (RCT) } \\
\text { RT: } \\
\text { FU: } 48 \text { weeks } \\
\text { Participants: } 40 \text { (20 with vitD; } 20 \\
\text { with Placebo) } \\
\text { Female/Male: } 26 / 14 \text { (VitD 12/8; } \\
\text { Placebo 14/6) } \\
\text { Mean Age (SD): VitD } 37.6 \text { (9.6); } \\
\text { placebo } 38.5 \text { (7.8) } \\
\text { Diagnosis MS: McDonald's criteria } \\
\text { Clinical Status: Being relapse-free } \\
\text { for } 30 \text { days prior to participation, } \\
\text { having had their first clinical event } \\
\text { in the previous } 5 \text { years, not active in } \\
\text { the } 30 \text { days prior to inclusion, RR } \\
\text { MS, treated with interferon- } \beta 1 \alpha .\end{array}$ & $\begin{array}{l}\text { Analyzed fluid: serum } \\
\text { Analythical methodology: } \\
\text { radioimmunoassay } \\
\text { Vit. D median (Q1-Q3) (nmol/L): } \\
\text { baseline control 53 (Q1-Q3 43-63), } \\
\text { baseline case 58 (Q1-Q3 38-82); } \\
\text { endpoint: control 61 (44-84), case } \\
226 \text { (159-250) } \\
\text { Vit. D status: NA } \\
\text { Dietary vit. D intake: NA } \\
\text { NB: D3 supplementation in the vit } \\
\text { D group cholecalciferol dosed at } \\
7000 \text { IU / day in the first } 4 \text { week, } \\
\text { followed by } 14,000 \text { IU/day up to } \\
\text { endpoint. }\end{array}$ & $\begin{array}{l}\text { Evaluation of } \\
\text { depressive } \\
\text { symptoms: } \\
\text { Hospital Anxiety } \\
\text { and Depression } \\
\text { Scale (HADS-D) } \\
\text { Depressive } \\
\text { symptoms severity: } \\
\text { baseline control 3.0 } \\
\text { (Q1-Q3 2.0-7.0), } \\
\text { baseline case 4.0 } \\
\text { (Q1-Q3 2.0-5.0) }\end{array}$ & $\begin{array}{l}\text { Sun exposure: } \\
\text { participants } \\
\text { quantified how } \\
\text { much time they } \\
\text { spent in the sun } \\
\text { during weekends } \\
\text { and holidays in } \\
\text { the current and } \\
\text { preceding } \\
\text { 3-month intervals } \\
\text { using validated } \\
\text { questions } \\
\text { Fatigue } \\
\text { Diagnosis: The } \\
\text { Fatigue Severity } \\
\text { Scale (FSS) } \\
\text { Inflammation } \\
\text { Status: } \\
\text { assessment of } \\
\text { TNF } \alpha \text { and IL-10 } \\
\text { concentrations }\end{array}$ & $\begin{array}{l}\text { Aim: Exploring the effect of high dose vitamin D3 supplementation on } \\
\text { depressive symptoms in MS. Fatigue was assessed as a potential } \\
\text { confounder. } \\
\text { Results: High dose vitamin D supplementation does not decrease } \\
\text { depression and fatigue scores. } \\
\text { Effect size: non significant, effect size not reported } \\
\text { Controlled cofounders: HADS-D at baseline and FSS at T1 } \\
\text { Quality assessment (Cochrane Risk of Bias 2.0): } \\
\text { Randomization process: Some concerns } \\
\text { Deviations from intended interventions: High risk } \\
\text { Mising outcome data: Some concerns } \\
\text { Measurement of the outcome: High risk } \\
\text { Selection of the reported result: Some concerns } \\
\text { Overall Bias: High risk }\end{array}$ \\
\hline
\end{tabular}




\subsection{What Is the Association between Circulating Levels of Vitamin D and Symptoms} of Depression?

In our scoping-review we found that four studies suggested a potential beneficial association between vitamin D circulating levels of vitamin D and depressive symptoms in pwMS. For instance, Ashtari et al. [43] found that low serum vitamin D level was inversely associated with depression scores. Similar results were reported by the studies of Knippenber et al. [48] and Silva et al. [45]. El Salem 2021 et al. [26] found a significant correlation between serum vitamin $\mathrm{D}$ level and scores of depression scales in males but not in females. Other studies $[44,46]$ did not find a significant association between vitamin D levels and depression.

\subsection{What Is the Association between Vitamin D Supplementation or Sun Exposure on Depression Symptoms?}

Freitas et al. [44] reported that sun exposure might have had an impact on depressive symptoms. In particular, they suggested that people with more depressive symptoms would refrain from outdoor activities, and this could account for the apparent relationship between sun exposure and depression. Knippenberg et al. [51] found that vitamin D levels were inversely associated with depression scores, but they related their results to sun exposure and not to vitamin D status.

Regarding the effect of vitamin D supplementation on depressive symptoms in pwMS, Taylor 2014 et al. [47] and Taylor 2018 [49] found that vitamin D supplementation reduced depression risk for pwMS. These findings were confirmed by Kotb et al. study [50]. The authors reported that lower vitamin D levels were associated with higher depressive scores and suggested that vitamin D replacement could have improved depressive symptoms in patients with RRMS. In the randomized controlled study by Rolf et al. [52], vitamin D supplementation did not affect depressive symptoms in the study group compared to the placebo group.

\subsection{Study Design Cross-Sectional Studies}

Ashtari et al. [43] assessed the relationship between vitamin D status, fatigue, and depressive symptoms. They referred to serum vitamin $\mathrm{D}$ as normal or low and they found that a low serum vitamin D level was inversely associated with depression scores. Their findings in this regard were similar to the studies of Knippenber et al. [48] and Silva et al. [45]. In their study, Knippenberg et al. [48] observed that vitamin D status was negatively correlated with depressive symptoms. However, after controlling for age, Expanded Disability Status Score (EDSS), and fatigue the statistical significance was lost. Silva et al. [45] evaluated the relationship between serum vitamin D levels, cognitive impairment, depression and fatigue. The authors found potential correlation between low vitamin $\mathrm{D}$ levels and depression, fatigue, and cognitive impairment. Freitas et al. [44] reported low vitamin D levels in $42.6 \%$ of MS patients, no correlation between vitamin D levels and depression was found. Tiu et al. [46] investigated the relationship between vitamin D status, depression and self-reported impact of disease. They did not find any significant association between low vitamin D levels and depression. El Salem 2021 et al. [26] observed a significant correlation between serum vitamin D levels and scores of depression scales in males but not in females. Taylor 2014 et al. [47] studied the association between modifiable lifestyle factors and risk of depression in MS patients. They found that vitamin D supplementation reduced depression risk for people with MS. In their follow-up study, the authors investigated whether modifiable lifestyle factors were associated with depression over 2.5 years of follow-up. Their results showed that vitamin D supplementation was associated with a reduction in the risk of developing depression [49].

Longitudinal Studies

The prospective observational study by Kotb et al. [50] explored whether depression in MS was related to vitamin D deficiency and whether the replacement of vitamin D would 
have improved depressive symptoms in this group of patients. Their results showed that lower vitamin $\mathrm{D}$ levels were associated with higher depressive scores and that vitamin $\mathrm{D}$ replacement could improve depressive symptoms in patients with RRMS. The prospective longitudinal study by Knippenberg et al. [51] examined the associations between sun exposure, serum vitamin D, depression, anxiety, fatigue, and cognition in MS patients. The authors found that vitamin D levels were inversely associated with depression scores, but that this was not significant after adjustment for patient-reported sun exposure. The randomized controlled study by Rolf et al. [52] explored the effect of high-dose vitamin D supplementation on depressive symptoms and on pro- and anti-inflammatory cytokine secretion by peripheral blood mononuclear cells (PBMC), PBMC and CD8+ T cells. Although a significant decrease in depressive symptoms was observed within the vitamin D supplementation arm, this reduction was not significantly different from the decrease seen in the placebo group. After 48 weeks of treatment with vitamin D supplementation, there was no reduction in the depressive symptoms or related pro- and anti-inflammatory cytokine balances secreted by stimulated leukocytes and CD8+ T cells.

\section{Discussion}

This study was carried out to provide an updated summary of previous evidence on the association between vitamin D status and depression in pwMS. Previous systematic reviews and meta-analysis on vitamin D and MS have focused on the impact of vitamin $\mathrm{D}$ on fatigue, disability status, and relapsing symptoms $[30,53]$ evaluating the effects on depressive symptoms as a secondary outcome. To our knowledge, this is the first review that has investigated the relationship between vitamin $\mathrm{D}$ and depressive symptoms in pwMS. Among the studies correlating vitamin D serum level with depressive symptoms, Ashtari et al. [43], Knippenberg et al. [48], Silva et al. [45], and El Salem 2021 et al. [26], found that low serum circulating levels of vitamin $\mathrm{D}$ were inversely associated with depression scale scores. The reported effect sizes ranged between small and medium values. The other two correlational studies revealed a potential negative link between vitamin D levels and depression $[44,46]$

Studies on vitamin D supplements used as a treatment for depression in MS present conflicting results. Taylor et al. [47,49] showed that vitamin D supplementation was associated with a change in the risk for depression. However, their data may be limited by the study design. Indeed, they conducted an online survey with a heterogenous sample between the time points. Kotb et al. [50] found that vitamin D supplementation improved depressive symptoms in patients with RRMS. However, they reported data on a relatively small number of patients and the authors explained that the sample was too small to confirm the existence of such important association. The other studies investigating the role of vitamin D supplementation reported non-significant effect sizes. In particular, the only available RCT explored the effect of vitamin D supplementation on depressive symptoms and anti-inflammatory cytokine levels [52]. The data from this RCT study does not support the role of vitamin D in affecting depressive symptoms. Their inconclusive finding should be considered in light of the original aim of their project. The study participants presented a median Hospital Anxiety and Depression Scale (HADS) of 3 and 4 for active and control arms, respectively. Therefore, if a high evidence output might answer our scoping review question, their findings are not easily generalizable to patients with depression and MS in comorbidity [54].

Considering potential confounding factors, it appears that sun exposure, age, sex, degree of disability, and fatigue represent the most common factors, which could have influenced results of the studies included. However, Ashtari et al. [43] and Knippenberg et al. [51] reported a significant correlation between vitamin D status and depressive symptoms but not between vitamin $\mathrm{D}$ and fatigue. Moreover, Ashtari et al., did not consider the seasonal variation of vitamin D and they did not consider the other confounding factors [43]. Meanwhile, Knippenberg et al. found no significance after controlling for EDSS, age, and fatigue [48]. Regarding the disability status in MS patients, one recent study 
found that vitamin D supplementation had no effects on EDSS [55]. These findings are confirmed by a meta-analysis by Hanaei et al. [53]. In another study by Knippenberg et al., the authors reported conflicting results when they compared serum vitamin D levels as a continuous or categorical variable. They found a significant negative correlation with depressive symptoms only when vitamin D serum levels were higher than $80 \mathrm{nmol} / \mathrm{L}$. Vitamin D deficiency was associated with depressive symptoms, but sun exposure had a moderating effect on them [51]. Moreover, in their study, Tiu et al. in their study found no significant associations between low vitamin D levels and depression. Additionally, vitamin D deficiency was associated with worse physical and psychological outcome measures [46] Yet, El-Salem et al. [26] reported on the one hand a stronger negative correlation between serum vitamin D and depression scores in males, and on the other hand, they found that disability measures, such as EDSS, were correlated with females' vitamin D levels in females only.

We do not know the exact mechanisms behind the association between vitamin $\mathrm{D}$ deficiency and depression, but there are several theories. According to Geng et al., it has been shown that a deficiency of vitamin D could affect the synthesis of serotonin, dopamine, and noradrenaline in the hippocampus, substantia nigra and prefrontal cortex [56]. Other authors have shown that vitamin D is involved in controlling the expression of those genes that are responsible for maintaining both $\mathrm{Ca}^{2+}$ and reactive oxygen species (ROS) homeostasis [57]. Moreover, vitamin D deficiency was linked to high levels of $\mathrm{Ca}^{2+}$ and ROS, which might affect neuronal cells and explain the link with depression. Indeed, an increase in the formation of ROS may exert a profound effect on neuronal function and has been observed in depression [32].

The mechanism by which vitamin $\mathrm{D}$ antidepressant therapeutic effect might be mediated remains uncertain. Both biological and psychological factors are thought to play a role in the etiology of depression in MS. A growing body of evidence highlights the role of a possible imbalance in pro- and anti-inflammatory cytokines in the development of depression in MS [58-60]. Moreover, the pro-inflammatory immunological changes suggested in depression may be linked to the disease activity, thereby worsening MS symptoms [61]. Preclinical literature supports the role of vitamin D on brain function. It has been demonstrated that vitamin $\mathrm{D}$ increases the brain concentration of brain-derived neurotrophic factor (BDNF) [62], which is a neurotrophic factor that modulates plasticity, mood, and the effects of antidepressants [63]. In addition, vitamin D modulates several brain areas, including the prefrontal cortex [64], which is an important brain area in the pathogenesis of depression and a target of newly non-pharmacological treatments $[65,66]$. Lastly, vitamin D is a strong regulator of brain morphology and neurogenesis $[67,68]$. Previous studies indicate that animal models of both depression and MS show abnormal hippocampal neurogenesis and neuronal morphology [69] and that morphological brain plasticity (neurogenesis, dendritic spine density, and dendritic complexity) is modulated by antidepressant treatments [70,71]. On the whole, preclinical results point to a brain plasticity modulating activity of vitamin $\mathrm{D}$ and support its role in the pathogenesis of depression in pwMS. Furthermore, regarding depressive symptoms in patients with MS and in depression in general, the data on Vitamin D still appear still unclear [58]. Indeed, the differences among the studies may be related in part to the small sample size, the seasonal variation of vitamin $\mathrm{D}$ and the different depressive symptoms scores at baseline. Moreover, the cross-sectional design studies we included may only establish an association but not causality. We should emphasize the fact that some studies did not perform all assessments on the same day and most of the studies did not examine confounding factors, such as sex differences. This is of pivotal importance as previous studies indicate sex difference in perceived stress [72], which was previously associated with depressive symptoms in MS [73]. This study presents several limitations: the search did not take into consideration grey literature or non-English studies due to lack of resources. In addition, we did not register a study protocol. The main reason was the exploratory nature of the present work. The strengths of the study are the novelty of the systematic approach in terms of search 
and data extraction, the evaluation of confounding factors, and the evidence gradient that underlines the need for further research.

\section{Conclusions}

The scoping review identified eleven studies that provided information on the role of vitamin D in affecting depressive symptoms in MS. Although some studies suggested a potential beneficial correlation between vitamin $\mathrm{D}$ and depression in pwMS, the results from cross-sectional and prospective studies are inconclusive. Future RCT studies on vitamin $\mathrm{D}$ and depressive symptoms in MS should include patients with actual depressive symptoms scores at baseline and should consider confounding factors, such as sun exposure and seasonal variation of vitamin D.

Author Contributions: Conceptualization: C.C. and A.R.; methodology: A.R., A.C. and A.N. writing-original draft preparation: C.C., A.C. and A.R., writing-review and editing: C.M., L.M., F.B., E.A. All authors have read and agreed to the published version of the manuscript.

Funding: This research received no external funding.

Institutional Review Board Statement: Not applicable.

Informed Consent Statement: Not applicable.

Conflicts of Interest: The authors declare no conflict of interest.

\section{References}

1. Dobson, R.; Giovannoni, G. Multiple sclerosis-A review. Eur. J. Neurol. 2019, 26, 27-40. [CrossRef] [PubMed]

2. Walton, C.; King, R.; Rechtman, L.; Kaye, W.; Leray, E.; Marrie, R.A.; Robertson, N.; La Rocca, N.; Uitdehaag, B.; van der Mei, I.; et al. Rising prevalence of multiple sclerosis worldwide: Insights from the Atlas of MS, third edition. Mult. Scler. J. 2020, 26, 1816-1821. [CrossRef] [PubMed]

3. D'Amico, E.; Patti, F.; Zanghì, A.; Chisari, C.G.; Lo Fermo, S.; Zappia, M. Late-onset and young-onset relapsing-remitting multiple sclerosis: Evidence from a retrospective long-term follow-up study. Eur. J. Neurol. 2018, 25, 1425-1431. [CrossRef] [PubMed]

4. Thompson, A.J.; Banwell, B.L.; Barkhof, F.; Carroll, W.M.; Coetzee, T.; Comi, G.; Correale, J.; Fazekas, F.; Filippi, M.; Freedman, M.S.; et al. Diagnosis of multiple sclerosis: 2017 revisions of the McDonald criteria. Lancet Neurol. 2018, 17, 162-173. [CrossRef]

5. McGinley, M.P.; Goldschmidt, C.H.; Rae-Grant, A.D. Diagnosis and Treatment of Multiple Sclerosis: A Review. JAMA 2021, 325, 765-779. [CrossRef]

6. D'Amico, E.; Zanghì, A.; Serra, A.; Murabito, P.; Zappia, M.; Patti, F.; Cocuzza, S. Management of dysphagia in multiple sclerosis: Current best practice. Expert Rev. Gastroenterol. Hepatol. 2019, 13, 47-54. [CrossRef]

7. D'Amico, E.; Patti, F.; Zanghì, A.; Zappia, M. A Personalized Approach in Progressive Multiple Sclerosis: The Current Status of Disease Modifying Therapies (DMTs) and Future Perspectives. Int. J. Mol. Sci. 2016, 17, 1725. [CrossRef]

8. Zanghì, A.; Cimino, S.; Urzì, D.; Privitera, S.; Zagari, F.; Lanza, G.; Patti, F.; D'Amico, E. Pharmacotherapeutic management of lower urinary tract symptoms in Multiple Sclerosis patients. Expert Opin. Pharmacother. 2020, 21, 1449-1454. [CrossRef]

9. Alvino, B.; Arianna, F.; Assunta, B.; Antonio, C.; Emanuele, D.; Giorgia, M.; Leonardo, S.; Daniele, S.; Renato, D.; Buscarinu, M.C.; et al. Prevalence and predictors of bowel dysfunction in a large multiple sclerosis outpatient population: An Italian multicenter study. J. Neurol. 2021. Online ahead of print. [CrossRef]

10. Biernacki, T.; Sandi, D.; Kincses, Z.T.; Füvesi, J.; Rózsa, C.; Mátyás, K.; Vécsei, L.; Bencsik, K. Contributing factors to health-related quality of life in multiple sclerosis. Brain Behav. 2019, 9, e01466. [CrossRef]

11. Solaro, C.; Gamberini, G.; Masuccio, F.G. Depression in Multiple Sclerosis: Epidemiology, Aetiology, Diagnosis and Treatment. CNS Drugs 2018, 32, 117-133. [CrossRef]

12. Feinstein, A.; Magalhaes, S.; Richard, J.F.; Audet, B.; Moore, C. The link between multiple sclerosis and depression. Nat. Rev. Neurol. 2014, 10, 507-517. [CrossRef] [PubMed]

13. Kobelt, G.; Thompson, A.; Berg, J.; Gannedahl, M.; Eriksson, J.; Group, M.S.; European Multiple Sclerosis, P. New insights into the burden and costs of multiple sclerosis in Europe. Mult. Scler. 2017, 23, 1123-1136. [CrossRef] [PubMed]

14. Pompili, M.; Forte, A.; Palermo, M.; Stefani, H.; Lamis, D.A.; Serafini, G.; Amore, M.; Girardi, P. Suicide risk in multiple sclerosis: A systematic review of current literature. J. Psychosom. Res. 2012, 73, 411-417. [CrossRef] [PubMed]

15. Patti, F.; Leone, C.; D'Amico, E. Treatment options of cognitive impairment in multiple sclerosis. Neurol. Sci. 2010, 31 (Suppl. 2), 265-269. [CrossRef]

16. Zanghì, A.; D'Amico, E.; Lo Fermo, S.; Patti, F. Exploring polypharmacy phenomenon in newly diagnosed relapsing-remitting multiple sclerosis: A cohort ambispective single-centre study. Ther. Adv. Chronic Dis. 2021, 12, 2040622320983121. [CrossRef]

17. Zanghì, A.; D'Amico, E.; Patti, F. Immunosuppression in relapsing remitting multiple sclerosis: Moving towards personalized treatment. Expert Rev. Neurother. 2020, 20,771-782. [CrossRef] 
18. D’Amico, E.; Chisari, C.G.; Arena, S.; Zanghì, A.; Toscano, S.; Lo Fermo, S.; Maimone, D.; Castaing, M.; Sciacca, S.; Zappia, M.; et al. Cancer Risk and Multiple Sclerosis: Evidence From a Large Italian Cohort. Front. Neurol. 2019, 10, 337. [CrossRef]

19. D'Amico, E.; Leone, C.; Zanghì, A.; Fermo, S.L.; Patti, F. Lateral and escalation therapy in relapsing-remitting multiple sclerosis: A comparative study. J. Neurol. 2016, 263, 1802-1809. [CrossRef]

20. Munger, K.L.; Hongell, K.; Åivo, J.; Soilu-Hänninen, M.; Surcel, H.M.; Ascherio, A. 25-Hydroxyvitamin D deficiency and risk of MS among women in the Finnish Maternity Cohort. Neurology 2017, 89, 1578-1583. [CrossRef]

21. Yadav, V.; Narayanaswami, P. Complementary and alternative medical therapies in multiple sclerosis-the American Academy of Neurology guidelines: A commentary. Clin. Ther. 2014, 36, 1972-1978. [CrossRef] [PubMed]

22. Mojaverrostami, S.; Bojnordi, M.N.; Ghasemi-Kasman, M.; Ebrahimzadeh, M.A.; Hamidabadi, H.G. A Review of Herbal Therapy in Multiple Sclerosis. Adv. Pharm. Bull. 2018, 8, 575-590. [CrossRef] [PubMed]

23. Concerto, C.; Boo, H.; Hu, C.; Sandilya, P.; Krish, A.; Chusid, E.; Coira, D.; Aguglia, E.; Battaglia, F. Hypericum perforatum extract modulates cortical plasticity in humans. Psychopharmacology 2018, 235, 145-153. [CrossRef] [PubMed]

24. Concerto, C.; Infortuna, C.; Muscatello, M.R.A.; Bruno, A.; Zoccali, R.; Chusid, E.; Aguglia, E.; Battaglia, F. Exploring the effect of adaptogenic Rhodiola Rosea extract on neuroplasticity in humans. Complement. Ther. Med. 2018, 41, 141-146. [CrossRef]

25. Mineo, L.; Concerto, C.; Patel, D.; Mayorga, T.; Paula, M.; Chusid, E.; Aguglia, E.; Battaglia, F. Valeriana officinalis Root Extract Modulates Cortical Excitatory Circuits in Humans. Neuropsychobiology 2017, 75, 46-51. [CrossRef]

26. El-Salem, K.; Khalil, H.; Al-Sharman, A.; Al-Mistarehi, A.H.; Yassin, A.; Alhayk, K.A.; Qawasmeh, M.A.; Bashayreh, S.Y.; Kofahi, R.M.; Obeidat, A.Z. Serum vitamin d inversely correlates with depression scores in people with multiple sclerosis. Mult. Scler. Relat. Disord. 2021, 48, 102732. [CrossRef]

27. Pierrot-Deseilligny, C.; Souberbielle, J.C. Vitamin D and multiple sclerosis: An update. Mult. Scler. Relat. Disord. 2017, 14, 35-45. [CrossRef]

28. Peelen, E.; Knippenberg, S.; Muris, A.H.; Thewissen, M.; Smolders, J.; Tervaert, J.W.; Hupperts, R.; Damoiseaux, J. Effects of vitamin D on the peripheral adaptive immune system: A review. Autoimmun. Rev. 2011, 10, 733-743. [CrossRef]

29. Haroon, E.; Raison, C.L.; Miller, A.H. Psychoneuroimmunology meets neuropsychopharmacology: Translational implications of the impact of inflammation on behavior. Neuropsychopharmacology 2011, 37, 137-162. [CrossRef]

30. Yuan, X.; Guo, L.; Jiang, C.; Yang, X.; Huang, J. The Effect of Different Administration Time and Dosage of Vitamin D Supplementation in Patients with Multiple Sclerosis: A Meta-Analysis of Randomized Controlled Trials. Neuroimmunomodulation 2021, 28, 118-128. [CrossRef]

31. Zech, L.D.; Scherf-Clavel, M.; Daniels, C.; Schwab, M.; Deckert, J.; Unterecker, S.; Herr, A.S. Patients with higher vitamin D levels show stronger improvement of self-reported depressive symptoms in psychogeriatric day-care setting. J. Neural Transm. 2021, 128, 1233-1238. [CrossRef] [PubMed]

32. Berridge, M.J. Vitamin D and Depression: Cellular and Regulatory Mechanisms. Pharmacol. Rev. 2017, 69, 80-92. [CrossRef] [PubMed]

33. Elrayah, E.E.; Rogers, L.; Doggui, R.; Al-Jawaldeh, A. Vitamin D Insufficiency and Deficiency in the Eastern Mediterranean Region (EMR)-Misconceptions in Public Health Practice: A Scoping Review 2019-2020. J. Nutr. Sci. Vitaminol. 2020, 66, 389-395. [CrossRef]

34. Kubera, M.; Lin, A.H.; Kenis, G.; Bosmans, E.; van Bockstaele, D.; Maes, M. Anti-Inflammatory effects of antidepressants through suppression of the interferon-gamma/interleukin-10 production ratio. J. Clin. Psychopharmacol. 2001, 21, 199-206. [CrossRef] [PubMed]

35. Dogan-Sander, E.; Mergl, R.; Willenberg, A.; Baber, R.; Wirkner, K.; Riedel-Heller, S.G.; Röhr, S.; Schmidt, F.M.; Schomerus, G.; Sander, C. Inflammation and the Association of Vitamin D and Depressive Symptomatology. Nutrients 2021, 13, 1972. [CrossRef] [PubMed]

36. Jorde, R.; Sneve, M.; Figenschau, Y.; Svartberg, J.; Waterloo, K. Effects of vitamin D supplementation on symptoms of depression in overweight and obese subjects: Randomized double blind trial. J. Intern. Med. 2008, 264, 599-609. [CrossRef]

37. Ju, S.Y.; Lee, Y.J.; Jeong, S.N. Serum 25-hydroxyvitamin D levels and the risk of depression: A systematic review and meta-analysis. J. Nutr. Health Aging 2012, 17, 447-455. [CrossRef]

38. Tricco, A.C.; Lillie, E.; Zarin, W.; O’Brien, K.K.; Colquhoun, H.; Levac, D.; Moher, D.; Peters, M.D.J.; Horsley, T.; Weeks, L.; et al. PRISMA Extension for Scoping Reviews (PRISMA-ScR): Checklist and Explanation. Ann. Intern. Med. 2018, 169, 467-473. [CrossRef]

39. Wells, G.A.; Shea, B.; O'Connell, D.; Peterson, J.; Welch, V.; Losos, M.; Tugwell, P. The Newcastle-Ottawa Scale (NOS) for Assessing the Quality of Nonrandomised Studies in Meta-Analyses; Ottawa Hospital Research Institute: Ottawa, ON, Canada, 2000.

40. Herzog, R.; Álvarez-Pasquin, M.J.; Díaz, C.; Del Barrio, J.L.; Estrada, J.M.; Gil, Á. Are healthcare workers' intentions to vaccinate related to their knowledge, beliefs and attitudes? A systematic review. BMC Public Health 2013, 13, 154. [CrossRef]

41. National Institutes of Health. National Institutes of Health (NIH) Quality Assessment Tool for before-after (Pre-Post). Available online: https://www.nhlbi.nih.gov/health-topics/study-quality-assessment-tools (accessed on 16 December 2021).

42. Sterne, J.A.C.; Savovic, J.; Page, M.J.; Elbers, R.G.; Blencowe, N.S.; Boutron, I.; Cates, C.J.; Cheng, H.Y.; Corbett, M.S.; Eldridge, S.M.; et al. RoB 2: A revised tool for assessing risk of bias in randomised trials. BMJ 2019, 366, 14898. [CrossRef]

43. Ashtari, F.; Ajalli, M.; Shaygannejad, V.; Akbari, M.; Hovsepian, S. The relation between Vitamin D status with fatigue and depressive symptoms of multiple sclerosis. J. Res. Med. Sci. 2013, 18, 193-197. 
44. Freitas, R.A.C. Clinical Correlates Of Fatigue In Multiple Sclerosis. 2017. Available online: https://repositorio-aberto.up.pt/ handle/10216/104113 (accessed on 10 October 2021).

45. Silva, B.; Merino, A.; Eizaguirre, B.; Yastremiz, C.; Pita, C.; Alonso, R.; Vanotti, S.; Garcea, O. Relationship between Vitamin D levels and cognitive impairment, depression and fatigue in relapsing remitting multiple sclerosis patients. Mult. Scler. J. 2016, 22,266

46. Tiu, C.; Terecoasa, E.; Tiu, V.; Grigore, A.; Gavan, C.; Bajenaru, O. Patient-based outcome measures of the impact of disease in patients with multiple sclerosis and vitamin D deficiency. Mult. Scler. J. 2017, 23, 735-736.

47. Taylor, K.L.; Hadgkiss, E.J.; Jelinek, G.A.; Weiland, T.J.; Pereira, N.G.; Marck, C.H.; van der Meer, D.M. Lifestyle factors, demographics and medications associated with depression risk in an international sample of people with multiple sclerosis. BMC Psychiatry 2014, 14, 327. [CrossRef]

48. Knippenberg, S.; Bol, Y.; Damoiseaux, J.; Hupperts, R.; Smolders, J. Vitamin D status in patients with MS is negatively correlated with depression, but not with fatigue. Acta Neurol. Scand. 2010, 124, 171-175. [CrossRef] [PubMed]

49. Taylor, K.L.; Simpson, S., Jr.; Jelinek, G.A.; Neate, S.L.; De Livera, A.M.; Brown, C.R.; O'Kearney, E.; Marck, C.H.; Weiland, T.J. Longitudinal Associations of Modifiable Lifestyle Factors With Positive Depression-Screen Over 2.5-Years in an International Cohort of People Living With Multiple Sclerosis. Front. Psychiatry 2018, 9, 526. [CrossRef]

50. Kotb, M.A.; Kamal, A.M.; Aldossary, N.M.; Bedewi, M.A. Effect of vitamin D replacement on depression in multiple sclerosis patients. Mult. Scler. Relat. Disord. 2019, 29, 111-117. [CrossRef] [PubMed]

51. Knippenberg, S.; Damoiseaux, J.; Bol, Y.; Hupperts, R.; Taylor, B.V.; Ponsonby, A.L.; Dwyer, T.; Simpson, S.; van der Mei, I.A. Higher levels of reported sun exposure, and not vitamin D status, are associated with less depressive symptoms and fatigue in multiple sclerosis. Acta Neurol. Scand. 2014, 129, 123-131. [CrossRef] [PubMed]

52. Rolf, L.; Muris, A.H.; Bol, Y.; Damoiseaux, J.; Smolders, J.; Hupperts, R. Vitamin D3 supplementation in multiple sclerosis: Symptoms and biomarkers of depression. J. Neurol. Sci. 2017, 378, 30-35. [CrossRef]

53. Hanaei, S.; Sahraian, M.A.; Mohammadifar, M.; Ramagopalan, S.V.; Ghajarzadeh, M. Effect of Vitamin D Supplements on Relapse Rate and Expanded Disability Status Scale (EDSS) in Multiple Sclerosis (MS): A Systematic Review and Meta-Analysis. Int. J. Prev. Med. 2021, 12, 42. [CrossRef]

54. Honarmand, K.; Feinstein, A. Validation of the Hospital Anxiety and Depression Scale for use with multiple sclerosis patients. Mult. Scler. J. 2009, 15, 1518-1524. [CrossRef]

55. Maretzke, F.; Bechthold, A.; Egert, S.; Ernst, J.B.; Melo van Lent, D.; Pilz, S.; Reichrath, J.; Stangl, G.I.; Stehle, P.; Volkert, D.; et al. Role of Vitamin D in Preventing and Treating Selected Extraskeletal Diseases-An Umbrella Review. Nutrients 2020, 12, 969. [CrossRef]

56. Geng, C.; Shaikh, A.S.; Han, W.; Chen, D.; Guo, Y.; Jiang, P. Vitamin D and depression: Mechanisms, determination and application. Asia Pac. J. Clin. Nutr. 2019, 28, 689-694. [CrossRef]

57. Sassi, F.; Tamone, C.; D'Amelio, P. Vitamin D: Nutrient, Hormone, and Immunomodulator. Nutrients 2018, 10, 1656. [CrossRef] [PubMed]

58. Gowda, U.; Mutowo, M.P.; Smith, B.J.; Wluka, A.E.; Renzaho, A.M. Vitamin D supplementation to reduce depression in adults: Meta-analysis of randomized controlled trials. Nutrients 2015, 31, 421-429. [CrossRef]

59. Crupi, R.; Cambiaghi, M.; Spatz, L.; Hen, R.; Thorn, M.; Friedman, E.; Vita, G.; Battaglia, F. Reduced adult neurogenesis and altered emotional behaviors in autoimmune-prone B-cell activating factor transgenic mice. Biol. Psychiatry 2010, 67, 558-566. [CrossRef] [PubMed]

60. Crupi, R.; Cambiaghi, M.; Deckelbaum, R.; Hansen, I.; Mindes, J.; Spina, E.; Battaglia, F. n-3 fatty acids prevent impairment of neurogenesis and synaptic plasticity in B-cell activating factor (BAFF) transgenic mice. Prev. Med. 2012, 54, S103-S108. [CrossRef] [PubMed]

61. Gold, S.M.; Irwin, M.R. Depression and immunity: Inflammation and depressive symptoms in multiple sclerosis. Immunol. Allergy Clin. N. Am. 2009, 29, 309-320. [CrossRef]

62. Shirazi, H.A.; Rasouli, J.; Ciric, B.; Rostami, A.; Zhang, G.X. 1,25-Dihydroxyvitamin D3 enhances neural stem cell proliferation and oligodendrocyte differentiation. Exp. Mol. Pathol. 2015, 98, 240-245. [CrossRef]

63. Björkholm, C.; Monteggia, L.M. BDNF-A key transducer of antidepressant effects. Neuropharmacology 2015, 102, 72-79. [CrossRef] [PubMed]

64. Kuningas, M.; Mooijaart, S.P.; Jolles, J.; Slagboom, P.E.; Westendorp, R.G.; van Heemst, D. VDR gene variants associate with cognitive function and depressive symptoms in old age. Neurobiol. Aging 2009, 30, 466-473. [CrossRef] [PubMed]

65. Concerto, C.; Lanza, G.; Cantone, M.; Ferri, R.; Pennisi, G.; Bella, R.; Aguglia, E. Repetitive transcranial magnetic stimulation in patients with drug-resistant major depression: A six-month clinical follow-up study. Int. J. Psychiatry Clin. Pr. 2015, 19, 252-258. [CrossRef]

66. Concerto, C.; Al Sawah, M.; Chusid, E.; Trepal, M.; Taylor, G.; Aguglia, E.; Battaglia, F. Anodal transcranial direct current stimulation for chronic pain in the elderly: A pilot study. Aging Clin. Exp. Res. 2015, 28, 231-237. [CrossRef] [PubMed]

67. Zhu, Y.; Zhou, R.; Yang, R.; Zhang, Z.; Bai, Y.; Chang, F.; Li, L.; Sokabe, M.; Goltzman, D.; Miao, D.; et al. Abnormal neurogenesis in the dentate gyrus of adult mice lacking 1,25-dihydroxy vitamin D3 (1,25-(OH)2 D3). Hippocampus 2012, 22, 421-433. [CrossRef] 
68. Mineo, L.; Concerto, C.; Patel, D.; Mayorga, T.; Chusid, E.; Infortuna, C.; Aguglia, E.; Sarraf, Y.; Battaglia, F. Modulation of sensorimotor circuits during retrieval of negative Autobiographical Memories: Exploring the impact of personality dimensions. Neuropsychology 2018, 110, 190-196. [CrossRef]

69. Zhang, H.; Kim, Y.; Ro, E.J.; Ho, C.; Lee, D.; Trapp, B.D.; Suh, H. Hippocampal Neurogenesis and Neural Circuit Formation in a Cuprizone-Induced Multiple Sclerosis Mouse Model. J. Neurosci. 2020, 40, 447-458. [CrossRef]

70. Cambiaghi, M.; Crupi, R.; Bautista, E.L.; Elsamadisi, A.; Malik, W.; Pozdniakova, H.; Han, Z.; Buffelli, M.; Battaglia, F. The Effects of 1-Hz rTMS on Emotional Behavior and Dendritic Complexity of Mature and Newly Generated Dentate Gyrus Neurons in Male Mice. Int. J. Environ. Res. Public Health 2020, 17, 4074. [CrossRef]

71. Cambiaghi, M.; Cherchi, L.; Masin, L.; Infortuna, C.; Briski, N.; Caviasco, C.; Hazaveh, S.; Han, Z.; Buffelli, M.; Battaglia, F. High-frequency repetitive transcranial magnetic stimulation enhances layer II/III morphological dendritic plasticity in mouse primary motor cortex. Behav. Brain Res. 2021, 410, 113352. [CrossRef] [PubMed]

72. Infortuna, C.; Gratteri, F.; Benotakeia, A.; Patel, S.; Fleischman, A.; Muscatello, M.R.A.; Bruno, A.; Zoccali, R.A.; Chusid, E.; Han, Z.; et al. Exploring the Gender Difference and Predictors of Perceived Stress among Students Enrolled in Different Medical Programs: A Cross-Sectional Study. Int. J. Environ. Res. Public Health 2020, 17, 6647. [CrossRef]

73. Van Heugten, C.; Köhler, S.; Francke, M.; Bol, Y. The association between executive functioning, coping styles and depressive symptoms in patients with Multiple Sclerosis. Mult. Scler. Relat. Disord. 2019, 36, 101392. [CrossRef] [PubMed] 